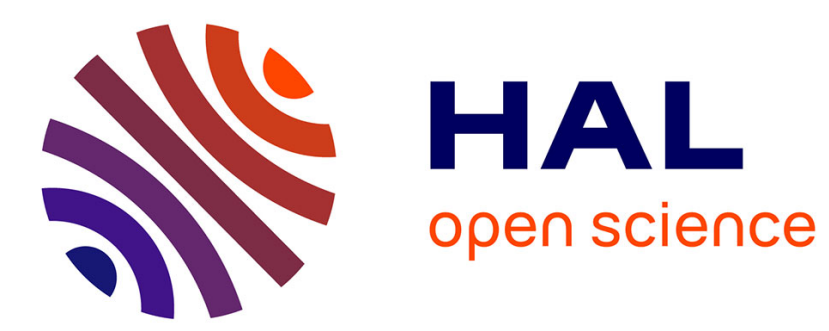

\title{
A closedness condition and its applications to DC programs with convex constraints
}

\author{
N. Dinh, T.T.A. Nghia, Guy Vallet
}

\section{To cite this version:}

N. Dinh, T.T.A. Nghia, Guy Vallet. A closedness condition and its applications to DC programs with convex constraints. Optimization, 2010, 59 (4), pp.541-560. hal-00221255

\section{HAL Id: hal-00221255 \\ https://hal.science/hal-00221255}

Submitted on 28 Jan 2008

HAL is a multi-disciplinary open access archive for the deposit and dissemination of scientific research documents, whether they are published or not. The documents may come from teaching and research institutions in France or abroad, or from public or private research centers.
L'archive ouverte pluridisciplinaire HAL, est destinée au dépôt et à la diffusion de documents scientifiques de niveau recherche, publiés ou non, émanant des établissements d'enseignement et de recherche français ou étrangers, des laboratoires publics ou privés. 


\title{
A closedness condition and its applications to DC programs with convex constraints *
}

\author{
N. Dinh ${ }^{\dagger} \quad$ T.T.A. Nghia ${ }^{\ddagger} \quad$ G. Vallet ${ }^{\S}$
}

\begin{abstract}
This paper concerns a closedness condition called (CC), requiring a convex function and a convex constrained system. This type of condition has played an important role in the study of convex optimization problems. Our aim is to establish several characterizations of this condition and to apply them to study minimizing problems involving a DC function under a cone-convex constraint and a set constraint. First, we establish several so-called "Toland-Fenchel-Lagrange" duality theorems. As consequences of these results, various versions of generalized Farkas lemmas in dual forms and optimality conditions for DC problem are obtained. A class of DC programs with semi-definite constraints is examined as an illustration. Most of these results are established under the (CC) and our paper serves as a link between several corresponding known ones published recently for DC programs and for convex programs.
\end{abstract}

Keywords: DC programs, closedness conditions, Farkas-Minkovski systems, Farkas lemmas, Fenchel-Lagrange duality, Toland- Fenchel-Lagrange duality.

AMS: 90C25, 90C26, 90C46, 49K30.

\section{Introduction}

Let us consider the DC optimization problem with convex constraints:

$$
\begin{aligned}
\text { inf } & f(x)-g(x) \\
\text { subject to } & x \in C, h(x) \in-S .
\end{aligned}
$$

Throughout this paper, we assume that: $X, Z$ are real locally convex Hausdorff topological vector spaces, $X^{*}$ (resp. $Z^{*}$ ) denotes the topological dual of $X$ (resp. $Z$ ), endowed with the weak*topologies; $C$ is a closed convex subset of $X ; f, g: X \rightarrow \mathbb{R} \cup\{+\infty\}$ are proper lower semicontinuous (l.s.c.) convex functions; $S$ is a closed convex cone of $Z$ (not necessarily with non-empty interior); and $h: X \rightarrow Z$ is an $S$-convex mapping, i.e.

$$
\forall u, v \in X, \forall t \in[0,1], \quad h(t u+(1-t) v)-t h(u)-(1-t) h(v) \in-S,
$$

such that $\lambda \circ h$ is l.s.c. for each $\lambda \in S^{+}$, the dual cone of $S$, denoted by:

$$
S^{+}:=\left\{\lambda \in Z^{*} \mid(\lambda, s) \geq 0, \text { for all } s \in S\right\}
$$

For convenience, for any $\lambda \in Z^{*}$, the composition of mappings $\lambda \circ h$ would be denoted by $\lambda h$.

We define, by convention, that $+\infty-(-\infty)=+\infty$.

\footnotetext{
* Parts of the work of the first author was realized during his visit at the Laboratory of Applied Mathematics, University of PAU to which he would like to express his sincere thanks for the hospitality he received.

†Department of Mathematics, International University, VNU-HCM, Ho Chi Minh City, Vietnam (ndinh@hcmiu.edu.vn). Questions, comments, or corrections to this document may be directed to that email address.

${ }^{\ddagger}$ Department of Mathematics and Computer Science, Ho Chi Minh City University of Pedagogy, HCM city, Vietnam (ttannghia@gmail.com).

$\S$ Laboratory of Applied Mathematics, UMR-CNRS 5142. University of PAU IPRA BP 1155, 64013 Pau Cedex, France (guy.vallet@univ-pau.fr).
} 
Recall that a function $p: X \rightarrow \mathbb{R} \cup\{+\infty\}$ is called a DC function, if it can be decomposed as a difference of two convex functions. Such class of functions covers the classes of convex functions, concave functions, and many other non-convex functions (see, e.g., [34, 36]).

The DC problem (P) has been studied by many authors since last decades (see [1], [20], [25], [28], [30], [32], [33], [34], [35] and references therein). Many real world problems possess this mathematical model (see [2], [3], [4], [5], [21]) and several numerical methods have been developed for this class of problems as well (see [3], [21], [36], and [34] for an overview).

It is well-known that for convex and DC optimization problems, a constraint qualification is essential ingredient for the Lagrange multiplier rule and for the duality theory. The well-known constraint qualifications for convex and DC optimization are often of the Slater types conditions (see $[28,29,14]$, for instance). However, these conditions are often not satisfied for many problems in applications. In the recent years, a condition called Farkas-Minkovski (or (FM) for short) that extends such a type of constraint qualifications, has been developed in [11, 12, 15, 16, 23, 24] for convex optimization problems. Moreover, in the cases when the cost functional is not continuous at any point in the feasible set, another condition called the closedness condition $[12,13,15,16]$ should be imposed. We are interested in a condition called (CC) [11] that replaces both of the mentioned conditions. We will give several characterizations of this condition. These characterizations will pave the way to derive strong duality and optimality conditions for the DC problem $(\mathrm{P})$.

Concerning the problem $(\mathrm{P})$, we consider the system

$$
\sigma:=\{x \in C, h(x) \in-S\}
$$

and the set of its solution $A$ which is the feasible set of $(\mathrm{P})$,

$$
A:=\{x \in X \mid x \in C, h(x) \in-S\}=C \cap h^{-1}(-S) .
$$

Throughout this paper, we assume that $A \cap \operatorname{dom} f \neq \emptyset$ and we denote by

$$
K:=\bigcup_{\lambda \in S^{+}} \operatorname{epi}(\lambda h)^{*}+\operatorname{epi} \delta_{C}^{*},
$$

where $\varphi^{*}$ stands for the conjugate of the function $\varphi$.

The system $\sigma$ is said to be Farkas-Minkovski (FM, in brief) when $K$ is weak*-closed.

The assumption that $\sigma$ is (FM) serves as a constraint qualification in the study of convex optimization problems. It was proposed in [23] (known as closed cone constraint qualification, (CCCQ)) and was used in $[9,15,16,24]$ to establish optimality conditions, duality and stability for convex (infinite) programming problems. Various sufficient conditions for (FM) were given in these mentioned papers. In particular, it was shown that the constraint qualification (FM) is strictly weaker than several generalized Slater type ones, and weaker than the Robinson type one stating that $\mathbb{R}_{+}[S+h(C)]$ is a closed subspace (see $[9,23]$ for more details).

Let us introduce the following closedness condition:

$$
\text { (CC) epi } f^{*}+K \text { is weak*-closed. }
$$

It involves the function $f$ and the system $\sigma$ and will play a crucial role in the sequel of this paper.

This closedness condition was proposed for the first time by R.S. Burachik and V. Jeyakumar in [11]. Then, it has been used in [27] to establish optimality conditions of Karush-Kuhn-Tucker form for convex cone-constrained programs. Several sufficient conditions for (CC) were given in [11] and [27]. A relaxed version, stating that epi $f^{*}+\operatorname{cl} K$ is weak ${ }^{*}$-closed, was introduced in [15] and [16] where this last condition, with the constraint qualification (FM) that imply (CC), were used to establish optimality conditions, duality and stability results, for convex infinite programs.

Let us mention too that the (CC) condition will be satisfied if $\sigma$ is (FM) and:

i) if on the one hand $f$ is continuous at least at one point in $A$ (see. $[11,16]$ ), or

ii) on the other hand, if cone $(\operatorname{dom} f-A)$ is a closed subspace of $X$. Indeed, if cone $(\operatorname{dom} f-A)=$ cone $\left(\operatorname{dom} f-\operatorname{dom} \delta_{A}\right)$ is a closed subspace, by [11]-Proposition 3.1, epi $f^{*}+\operatorname{epi} \delta_{A}^{*}$ is weak*-closed and so, thanks to (4), 


$$
\text { epi } f^{*}+\operatorname{epi} \delta_{A}^{*}=\operatorname{epi} f^{*}+\operatorname{cl} K=\operatorname{epi} f^{*}+K \text { is weak }{ }^{*} \text {-closed. }
$$

Note that if the set constraint " $x \in C$ " is absent, that is, if $\sigma:=\{h(x) \in-S\}$, the condition (CC) becomes: epif* $f^{*} \bigcup_{\lambda \in S^{+}} \operatorname{epi}(\lambda h)^{*}$ is weak*-closed.

In this paper, characterizations for (CC) in dual forms and in terms of approximate subdifferentials would be established. These results will serve as main tools to establish duality results and optimality conditions for $(\mathrm{P})$. We first consider various dual problems of $(\mathrm{P})$ which will be called "Toland-Fenchel-Lagrange" type dual problems (see [28] for example). It is, in some sense, a "combination" of Toland dual for DC problem in [32], Fenchel and Lagrange dual problems (see $[8,9,23])$. We establish several duality theorems which extend Laghdir's ones in [28] and various versions of generalized Farkas lemmas in dual forms for systems involving convex and DC functions are derived. Applied to convex systems or to convex programs, these results give strong Lagrange duality and Fenchel-Lagrange duality results, and extend known ones established for example in [8], $[9],[16],[17]$ and $[23]$.

Optimality conditions for DC problem are also obtained and a class of DC programs with semidefinite constraints is examined. Our results are obtained under the (CC) condition and by using its characterizations given in section 3. They serve as a link between several corresponding known ones published recently for DC programs and for convex programs.

The paper is organized as follows: in Section 2, we fix some notations and we recall results needed in the sequel of the paper. Characterizations of the condition (CC) are given in Section 3, and corollaries are derived from simple cases. In particular, characterizations of (FM) are also proposed, in particular to give a representation for the approximate normal cones to convex constrained sets. In Section 4, we establish several duality results of "Toland-Fenchel-Lagrange" type for the problem $(\mathrm{P})$, which extend some recent ones given in [28]. Corollaries for concrete classes of problems, including the classes of convex and concave programs, are obtained, which are compatible with the ones given in [8], [15],[16]. In Section 5, various versions of generalized Farkas lemma in dual forms are established for convex and DC functions, which cover the ones established in [8] for convex systems. In Section 6, optimality conditions for (P), as well as in the convex case, are proved. The problems of maximizing a convex function under convex constraints is also treated as a special case. Lastly, in Section 7, a class of DC programs with semi-definite constraints is considered as an illustration. Optimality conditions and duality results are obtained. These results extend the one obtained recently in $[17,24]$ for convex semi-definite problems.

\section{Preliminaries}

Let us fix some notations used in the sequel of the paper.

For a set $D \subset X$, the closure (resp. the convex hull) of $D$ will be denoted by $\operatorname{cl} D$ (with suitable topology) (resp. co $D$ ) and cone $D$ stands for the convex cone generated by $D$.

The indicator function of a set $D \subset X$ is defined by: $\delta_{D}(x)=0$ if $x \in D$ and $\delta_{D}(x)=+\infty$ else. Moreover, the support function $\sigma_{D}$ is given by $\sigma_{D}(u)=\sup _{x \in D} u(x)$.

Let $f: X \rightarrow \mathbb{R} \cup\{+\infty\}$ be a proper lower semicontinuous (l.s.c.) convex function. Then:

(i) The conjugate function of $f, f^{*}: X^{*} \rightarrow \mathbb{R} \cup\{+\infty\}$, is defined by

$$
f^{*}(v)=\sup \{v(x)-f(x) \mid x \in \operatorname{dom} f\},
$$

where the domain of $f$ is given by $\operatorname{dom} f:=\{x \in X \mid f(x)<+\infty\}$,

(ii) If $a \in \operatorname{dom} f$ then, following [22],

$$
\text { epi } f^{*}=\bigcup_{\epsilon \geq 0}\left\{(v, v(a)+\epsilon-f(a)) \mid v \in \partial_{\epsilon} f(a)\right\},
$$

where, for a given $\epsilon \geq 0$, the $\epsilon$-subdifferential of $f$ at $a \in \operatorname{dom} f, \partial_{\epsilon} f(a)$, is defined as the possibly empty closed convex set:

$$
\partial_{\epsilon} f(a)=\left\{v \in X^{*} \quad f(x)-f(a) \geq(v, x-a)-\epsilon, \forall x \in \operatorname{dom} f\right\} .
$$


(iii) If $\epsilon>0$ then $\partial_{\epsilon} f(a) \neq \emptyset$. Moreover, $\bigcap_{\epsilon>0} \partial_{\epsilon} f(a)=\partial f(a)$, where $\partial f(a)$ denotes the usual convex subdifferential of $f$ at $a$ (for more details, see [37]).

For a closed convex subset $D \subset X$ and an arbitrary $\epsilon \geq 0$, the $\epsilon$-normal cone to $D$ at a point $\bar{x} \in D$ is defined by (see $[19,20]$ )

$$
N_{\epsilon}(D, \bar{x}):=\partial_{\epsilon} \delta_{D}(\bar{x})=\left\{x^{*} \in X^{*} \mid\left(x^{*}, x-\bar{x}\right) \leq \epsilon, \forall x \in D\right\} .
$$

When $\epsilon=0, N_{0}(D, \bar{x})=N(D, \bar{x})$ is the normal cone to $D$ at $\bar{x}$ in the sense of convex analysis.

Following [11], it is worth noting that for two proper, l.s.c. convex functions $f$ and $g$,

$$
\text { epi }(f+g)^{*}=\operatorname{cl}\left(\text { epi } f^{*}+\text { epi } g^{*}\right) \text {. }
$$

Moreover, epi $f^{*}+$ epi $g^{*}$ is weak*-closed, if at least one of both functions $f$ or $g$ is continuous at some point of $\operatorname{dom} f \cap \operatorname{dom} g$ (see [11]).

Note that, if, as it is assumed, $h$ is a $S$-convex mapping such that $\lambda h$ is l.s.c. for each $\lambda \in S^{+}$, then $h^{-1}(-S):=\{x \in X \mid-h(x) \in S\}$ is convex and $A:=C \cap h^{-1}(-S)$ is closed. Moreover, $\cup_{\lambda \in S^{+}} \operatorname{epi}(\lambda h)^{*}$ is a convex cone (see [25]) and

$$
\operatorname{epi}_{A}^{*}=\mathrm{cl} K
$$

(note that the equality (4) has been proved in [16], [23] and [27]).

It is worth noticing that, if the mapping $h$ is sequentially lower semi-continuous in the sense given in [14], then $\lambda h$ is l.s.c for each $\lambda \in S^{+}$, provided that $X$ is metrizable (see [14]-Proposition 3.7).

Let us conclude this section by recalling some results on duality and optimality conditions for DC programs established by J. F. Toland in [32] and by J.B. Hiriart-Urruty in [19].

Lemma 2.1 [32] Let $X$ be a locally convex Hausdorff topological vector space with $X^{*}$ is its topological dual. Let further $F, G: X \rightarrow \mathbb{R} \cup\{+\infty\}$. Assume that $G$ is a proper, convex and l.s.c. function and $F$ is an arbitrary function. Then

$$
\inf _{x \in X}\{F(x)-G(x)\}=\inf _{u \in X^{*}}\left\{G^{*}(u)-F^{*}(u)\right\} .
$$

Lemma 2.2 [19] Let $X$ be a locally convex Hausdorff topological vector space and $F, G: X \rightarrow$ $\mathbb{R} \cup\{+\infty\}$ be a l.s.c, proper and convex functions. Then

(i) A point $a \in X$ is a global minimizer of the problem $\inf _{x \in X}\{F(x)-G(x)\}$ if and only if for any $\epsilon \geq 0, \partial_{\epsilon} G(a) \subset \partial_{\epsilon} F(a)$.

(ii) If $a \in X$ is a local minimizer of $\inf _{x \in X}\{F(x)-G(x)\}$ then $\partial G(a) \subset \partial F(a)$.

\section{Characterizations of the closedness condition (CC)}

In this section, we will establish necessary and sufficient conditions for the condition (CC). These conditions will be crucial in the sequel and they also deserve some attention for their independent interest. Then, characterizations of (FM) constraint qualification and of approximate normal cones to convex constrained sets are given at the end of the section.

Theorem 3.1 The following statements are equivalent:

(i) Condition $(C C)$ holds,

(ii) For all $x^{*} \in X^{*}$,

$$
\left(f+\delta_{A}\right)^{*}\left(x^{*}\right)=\min _{\lambda \in S^{+}} \min _{u, v \in X^{*}}\left[f^{*}(u)+(\lambda h)^{*}(v)+\delta_{C}^{*}\left(x^{*}-u-v\right)\right],
$$

(the infimum in the right hand side is attained at some $\lambda \in S^{+}$and $u, v \in X^{*}$ ),

(iii) For any $\bar{x} \in A \cap \operatorname{dom} f$ and each $\epsilon \geq 0$,

$$
\partial_{\epsilon}\left(f+\delta_{A}\right)(\bar{x})=\bigcup_{\lambda \in S^{+}} \bigcup_{\substack{\epsilon_{1}, \epsilon_{2}, \epsilon_{3} \geq 0 \\ \epsilon_{1}+\epsilon_{2}+\epsilon_{3}=\epsilon+\lambda h(\bar{x})}}\left\{\partial_{\epsilon_{1}} f(\bar{x})+\partial_{\epsilon_{2}} \lambda h(\bar{x})+N_{\epsilon_{3}}(C, \bar{x})\right\} .
$$


Proof. $\left[(i) \Longrightarrow\right.$ (ii)] Assume that (i) holds and consider any $x^{*} \in X^{*}$. Then, for all $u, v \in X^{*}$, $\lambda \in S^{+}$and $x \in A$, we have that

$$
\begin{aligned}
f^{*}(u) \geq(u, x)-f(x), & (\lambda h)^{*}(v) \geq(v, x)-\lambda h(x), \\
\delta_{C}^{*}\left(x^{*}-u-v\right) & \geq\left(x^{*}-u-v, x\right),
\end{aligned}
$$

which implies that $f^{*}(u)+(\lambda h)^{*}(v)+\delta_{C}^{*}\left(x^{*}-u-v\right) \geq\left(x^{*}, x\right)-f(x)-\lambda h(x) \geq\left(x^{*}, x\right)-f(x)$. Thus,

$$
f^{*}(u)+(\lambda h)^{*}(v)+\delta_{C}^{*}\left(x^{*}-u-v\right) \geq\left(x^{*}, x\right)-f(x)-\delta_{A}(x)
$$

for all $u, v \in X^{*}, \lambda \in S^{+}$and $x \in X$. This implies that

$$
f^{*}(u)+(\lambda h)^{*}(v)+\delta_{C}^{*}\left(x^{*}-u-v\right) \geq\left(f+\delta_{A}\right)^{*}\left(x^{*}\right),
$$

and hence,

$$
\inf _{\lambda \in S^{+}} \inf _{u, v \in X^{*}}\left[f^{*}(u)+(\lambda h)^{*}(v)+\delta_{C}^{*}\left(x^{*}-u-v\right)\right] \geq\left(f+\delta_{A}\right)^{*}\left(x^{*}\right) .
$$

If on the one hand $x^{*} \notin \operatorname{dom}\left(\mathrm{f}+\delta_{\mathrm{A}}\right)^{*}$, then $\left(f+\delta_{A}\right)^{*}\left(x^{*}\right)=+\infty$ and thanks to (8), (5) holds.

If on the other hand $x^{*} \in \operatorname{dom}\left(\mathrm{f}+\delta_{\mathrm{A}}\right)^{*}$, combining (3), (4) and $(\mathrm{CC})$, we get that

$$
\begin{aligned}
\operatorname{epi}\left(f+\delta_{A}\right)^{*} & =\operatorname{cl}\left(\text { epi } f^{*}+\operatorname{epi} \delta_{A}^{*}\right) \\
& =\operatorname{cl}\left(\operatorname{epi} f^{*}+\operatorname{cl} K\right)=\operatorname{cl}\left(\operatorname{epi} f^{*}+K\right) \\
& =\operatorname{epi} f^{*}+\cup_{\lambda \in S^{+}} \text {epi }(\lambda h)^{*}+\text { epi } \delta_{C}^{*} .
\end{aligned}
$$

Then, one has that

$$
\left(x^{*},\left(f+\delta_{A}\right)^{*}\left(x^{*}\right)\right) \in \operatorname{epi}\left(f+\delta_{A}\right)^{*}=\operatorname{epi} f^{*}+\cup_{\lambda \in S^{+}} \operatorname{epi}(\lambda h)^{*}+\text { epi } \delta_{C}^{*} .
$$

Thus, there exist $\lambda \in S^{+},(u, r) \in$ epi $f^{*},(v, s) \in$ epi $(\lambda h)^{*}$ and $(w, t) \in$ epi $\delta_{C}^{*}$, such that

$$
\left(x^{*},\left(f+\delta_{A}\right)^{*}\left(x^{*}\right)\right)=(u, r)+(v, s)+(w, t) .
$$

This implies that $u+v+w=x^{*}$ and that

$$
\left(f+\delta_{A}\right)^{*}\left(x^{*}\right) \geq f^{*}(u)+(\lambda h)^{*}(v)+\delta_{C}^{*}(w) .
$$

Thus, it comes that

$$
\left(f+\delta_{A}\right)^{*}\left(x^{*}\right) \geq f^{*}(u)+(\lambda h)^{*}(v)+\delta_{C}^{*}\left(x^{*}-u-v\right),
$$

and thanks to (8), (5) holds and the infimum is attained at some $\lambda \in S^{+}$and $u, v \in X^{*}$.

Thus, (ii) has been proved.

[(ii) $\Longrightarrow$ (iii)] Suppose that (ii) holds. Let $\epsilon$ be an arbitrary non-negative number and $x^{*}$ be a point in the set of the right-hand side of (6). Then, there exist $\lambda \in S^{+}, \epsilon_{1}, \epsilon_{2}, \epsilon_{3} \geq 0, u \in \partial_{\epsilon_{1}} f(\bar{x})$, $v \in \partial_{\epsilon_{2}}(\lambda h)(\bar{x})$ and $w \in N_{\epsilon_{3}}(C, \bar{x})$, such that $\epsilon_{1}+\epsilon_{2}+\epsilon_{3}=\epsilon+\lambda h(\bar{x})$ and $x^{*}=u+v+w$. Since

$$
\begin{array}{ll}
(u, x-\bar{x})-\epsilon_{1} \leq f(x)-f(\bar{x}) & \forall x \in X, \\
(v, x-\bar{x})-\epsilon_{2} \leq \lambda h(x)-\lambda h(\bar{x}) & \forall x \in X, \\
(w, x-\bar{x})-\epsilon_{3} \leq 0 & \forall x \in C,
\end{array}
$$

we get that $\left(x^{*}, x-\bar{x}\right)-\epsilon \leq f(x)-f(\bar{x})+\lambda h(x)$ for all $x \in C$. This yields

$$
\left(x^{*}, x-\bar{x}\right)-\epsilon \leq f(x)-f(\bar{x})+\lambda h(x) \leq f(x)-f(\bar{x}), \forall x \in A,
$$

and hence,

$$
\left(x^{*}, x-\bar{x}\right)-\epsilon \leq\left(f+\delta_{A}\right)(x)-\left(f+\delta_{A}\right)(\bar{x}), \forall x \in X,
$$

which proves that $x^{*} \in \partial_{\epsilon}\left(f+\delta_{A}\right)(\bar{x})$.

To prove the converse inclusion, we first observe that if $\epsilon=0$ and if $\partial\left(f+\delta_{A}\right)(\bar{x})=\emptyset$, then the converse inclusion holds trivially. So, we may suppose that $\partial_{\epsilon}\left(f+\delta_{A}\right)(\bar{x}) \neq \emptyset$ for all $\epsilon \geq 0$ (note that if $\epsilon>0$ then $\left.\partial_{\epsilon}\left(f+\delta_{A}\right)(\bar{x}) \neq \emptyset\right)$. 
Let $x^{*} \in \partial_{\epsilon}\left(f+\delta_{A}\right)(\bar{x})$. Since $\bar{x} \in \operatorname{dom}\left(f+\delta_{A}\right)$, it follows from (2) that

$$
\epsilon+\left(x^{*}, \bar{x}\right)-f(\bar{x})-\delta_{A}(\bar{x})=\epsilon+\left(x^{*}, \bar{x}\right)-f(\bar{x}) \geq\left(f+\delta_{A}\right)^{*}\left(x^{*}\right) .
$$

Thanks to (ii), there exist $\lambda \in S^{+}$and $u, v, w \in X^{*}$, such that $u+v+w=x^{*}$ and that $\left(f+\delta_{A}\right)^{*}\left(x^{*}\right)=$ $f^{*}(u)+(\lambda h)^{*}(v)+\delta_{C}^{*}(w)$. This and (11) give

$$
\epsilon+\left(x^{*}, \bar{x}\right)-f(\bar{x}) \geq f^{*}(u)+(\lambda h)^{*}(v)+\delta_{C}^{*}(w),
$$

which implies that $u \in \operatorname{dom} f^{*}, v \in \operatorname{dom}(\lambda h)^{*}$ and that $w \in \operatorname{dom} \delta_{C}^{*}$.

Since $\left(u, f^{*}(u)\right) \in \operatorname{epi} f^{*}$, by using (2) again, there exist $\epsilon_{1} \geq 0$ and $u^{\prime} \in \partial_{\epsilon_{1}} f(\bar{x})$, such that $\left(u, f^{*}(u)\right)=\left(u^{\prime},\left(u^{\prime}, \bar{x}\right)+\epsilon_{1}-f(\bar{x})\right)$.

This gives that $u=u^{\prime} \in \partial_{\epsilon_{1}} f(\bar{x})$ and that $\epsilon_{1}=f^{*}(u)-(u, \bar{x})+f(\bar{x})$.

Similarly, it comes that $v \in \partial_{\epsilon_{2}}(\lambda h)(\bar{x})$ and that $w \in \partial_{\epsilon_{3}^{\prime}} \delta_{C}(\bar{x})=N_{\epsilon_{3}^{\prime}}(C, \bar{x})$ where $\epsilon_{2}=(\lambda h)^{*}(v)-$ $(v, \bar{x})+\lambda h(\bar{x}) \geq 0$ and $\epsilon_{3}^{\prime}=\delta_{C}^{*}(w)-(w, \bar{x}) \geq 0$. Since $x^{*}=u+v+w$, it follows from (12) that

$$
\epsilon+\lambda h(\bar{x}) \geq\left\{f^{*}(u)-(u, \bar{x})+f(\bar{x})\right\}+\left\{(\lambda h)^{*}(v)-(v, \bar{x})+\lambda h(\bar{x})\right\}+\left\{\delta_{C}^{*}(w)-(w, \bar{x})\right\},
$$

which means that $\epsilon+\lambda h(\bar{x}) \geq \epsilon_{1}+\epsilon_{2}+\epsilon_{3}^{\prime}$.

Let $\epsilon_{3}=\epsilon+\lambda h(\bar{x})-\epsilon_{1}-\epsilon_{2}$, then $\epsilon+\lambda h(\bar{x})=\epsilon_{1}+\epsilon_{2}+\epsilon_{3}$ and $w \in N_{\epsilon_{3}^{\prime}}(C, \bar{x}) \subset N_{\epsilon_{3}}(N, \bar{x})$ as $\epsilon_{3}^{\prime} \leq \epsilon_{3}$. Consequently,

$$
x^{*}=u+v+w \in \bigcup_{\lambda \in S^{+}} \bigcup_{\substack{\epsilon_{1}, \epsilon_{2}, \epsilon_{3} \geq 0 \\ \epsilon_{1}+\epsilon_{2}+\epsilon_{3}=\epsilon+\lambda h(\bar{x})}}\left\{\partial_{\epsilon_{1}} f(\bar{x})+\partial_{\epsilon_{2}} \lambda h(\bar{x})+N_{\epsilon_{3}}(C, \bar{x})\right\} .
$$

Thus, (ii) implies (iii).

[(iii) $\Longrightarrow$ (i)] Suppose that (iii) holds and consider any $\left(x^{*}, r\right) \in \operatorname{cl}\left(\right.$ epi $\left.f^{*}+K\right)$. Then, thanks to (9), it comes that $\left(x^{*}, r\right) \in$ epi $\left(f+\delta_{A}\right)^{*}$. Since $\bar{x} \in \operatorname{dom}\left(f+\delta_{A}\right)$, it follows from (2) that $\epsilon \geq 0$ exists such that $x^{*} \in \partial_{\epsilon}\left(f+\delta_{A}\right)(\bar{x})$ and $r=\left(x^{*}, \bar{x}\right)-f(\bar{x})+\epsilon$.

Then, it comes from (iii) that there exist $\lambda \in S^{+}, u, v, w \in X^{*}$ and $\epsilon_{1}, \epsilon_{2}, \epsilon_{3} \geq 0$ satisfying $x^{*}=$ $u+v+w, \epsilon_{1}+\epsilon_{2}+\epsilon_{3}=\epsilon+\lambda h(\bar{x})$ with $u \in \partial_{\epsilon_{1}} f(\bar{x}), v \in \partial_{\epsilon_{2}} \lambda h(\bar{x})$ and $w \in N_{\epsilon_{3}}(C, \bar{x})$.

Set $s=(u, \bar{x})-f(\bar{x})+\epsilon_{1}, t=(v, \bar{x})-\lambda h(\bar{x})+\epsilon_{2}$ and $k=(w, \bar{x})+\epsilon_{3}$. Then, thanks to (2), one gets that $(u, s) \in$ epi $f^{*},(v, t) \in$ epi $(\lambda h)^{*}$ and $(w, k) \in$ epi $\delta_{C}^{*}$. Moreover, one has that

$$
\begin{aligned}
s+t+k & =(u, \bar{x})-f(\bar{x})+\epsilon_{1}+(v, \bar{x})-\lambda h(\bar{x})+\epsilon_{2}+(w, \bar{x})+\epsilon_{3} \\
& =\left(x^{*}, \bar{x}\right)-f(\bar{x})-\lambda h(\bar{x})+\epsilon_{1}+\epsilon_{2}+\epsilon_{3}=\left(x^{*}, \bar{x}\right)-f(\bar{x})+\epsilon=r .
\end{aligned}
$$

Thus,

$$
\left(x^{*}, r\right)=(u, s)+(v, t)+(w, k) \in \text { epi } f^{*}+\cup_{\lambda \in S^{+}} \text {epi }(\lambda h)^{*}+\text { epi } \delta_{C}^{*}=\text { epi } f^{*}+K,
$$

which proves that epi $f^{*}+K$ is weak* closed. In other words, (CC) holds and the theorem is completely proved.

Let us present in the following corollary, some useful results for the forth-coming demonstrations.

Corollary 3.1 Suppose that the condition (CC) holds. Then, for each $x^{*} \in X^{*}$,

$$
\left(f+\delta_{A}\right)^{*}\left(x^{*}\right)=\min _{\lambda \in S^{+}}\left(f+\lambda h+\delta_{C}\right)^{*}\left(x^{*}\right) .
$$

Proof. Let $x^{*} \in X^{*}$. Since (CC) holds, it follows from Theorem 3.1-ii) that $\bar{\lambda} \in S^{+}$and $u, v \in X^{*}$ exist such that

$$
\left(f+\delta_{A}\right)^{*}\left(x^{*}\right)=f^{*}(u)+(\bar{\lambda} h)^{*}(v)+\delta_{C}^{*}\left(x^{*}-u-v\right) .
$$

Thus, for each $x \in X$, one gets that

$$
\begin{aligned}
\left(f+\delta_{A}\right)^{*}\left(x^{*}\right) & \geq(u, x)-f(x)+(v, x)-\bar{\lambda} h(x)+\left(x^{*}-u-v, x\right)-\delta_{C}(x) \\
& \geq\left(x^{*}, x\right)-\left(f+\bar{\lambda} h+\delta_{C}\right)(x)
\end{aligned}
$$


which implies that

$$
\left(f+\delta_{A}\right)^{*}\left(x^{*}\right) \geq\left(f+\bar{\lambda} h+\delta_{C}\right)^{*}\left(x^{*}\right) .
$$

On the other hand, for each $\lambda \in S^{+}$,

$$
\begin{aligned}
\left(f+\lambda h+\delta_{C}\right)^{*}\left(x^{*}\right) & =\sup _{x \in C}\left\{\left(x^{*}, x\right)-(f+\lambda h)(x)\right\} \\
& \geq \sup _{x \in A}\left\{\left(x^{*}, x\right)-(f+\lambda h)(x)\right\} \\
& \geq \sup _{x \in A}\left\{\left(x^{*}, x\right)-f(x)\right\}=\left(f+\delta_{A}\right)^{*}\left(x^{*}\right) .
\end{aligned}
$$

Combining (14) and (15), we get that

$$
\left(f+\delta_{A}\right)^{*}\left(x^{*}\right)=\min _{\lambda \in S^{+}}\left(f+\lambda h+\delta_{C}\right)^{*}\left(x^{*}\right)=\left(f+\bar{\lambda} h+\delta_{C}\right)^{*}\left(x^{*}\right),
$$

and the proof is complete.

Note that in the absence of the set constraint " $x \in C$ " (i.e., $C=X$ ), characterizations of $(\mathrm{CC})$ are given in the following corollary, whose proofs are the same as those of Theorem 3.1 and hence, will be omitted.

Corollary 3.2 Suppose that $C=X$. The following statements are equivalent:

(i) Condition (CC) holds,

(ii) for each $x^{*} \in X^{*},\left(f+\delta_{A}\right)^{*}\left(x^{*}\right)=\min _{\lambda \in X^{*}} \min _{u \in X^{*}}\left\{f^{*}(u)+(\lambda h)^{*}\left(x^{*}-u\right)\right\}$,

(iii) For each $\epsilon \geq 0$ and each $\bar{x} \in \operatorname{dom} f \cap h^{-1}(-S)$,

$$
\partial_{\epsilon}\left(f+\delta_{A}\right)(\bar{x})=\bigcup_{\lambda \in S^{+}} \bigcup_{\substack{\epsilon_{1}, \epsilon_{2} \geq 0 \\ \epsilon_{1}+\epsilon_{2}=\epsilon+\lambda h(\bar{x})}}\left\{\partial_{\epsilon_{1}} f(\bar{x})+\partial_{\epsilon_{2}} \lambda h(\bar{x})\right\} .
$$

Let us give now representations of the approximate normal cones to the convex constrained set $A$. These representations are useful in optimization problems when one needs to establish the optimality conditions or the characterization of approximate solutions of the mentioned problems. Note that this result gives also a characterization of the (FM) constraint qualification for the system $\sigma$. For more details and other results on the approximate normal cones, see [18].

Corollary 3.3 The following statements are equivalent:

(i) The system $\sigma$ is (FM),

(ii) For each $x^{*} \in X^{*}$,

$$
\sigma_{A}\left(x^{*}\right)=\min _{\lambda \in S^{+}} \min _{u \in X^{*}}\left[(\lambda h)^{*}(u)+\delta_{C}^{*}\left(x^{*}-u\right)\right]=\min _{\lambda \in S^{+}}\left[(\lambda h)^{*} \oplus \delta_{C}^{*}\right]\left(x^{*}\right),
$$

(iii) For any $a \in A$ and each $\epsilon \geq 0$,

$$
N_{\epsilon}(A, a)=\bigcup_{\lambda \in S^{+}} \bigcup_{\substack{\epsilon_{1}, \epsilon_{2} \geq 0 \\ \epsilon_{1}+\epsilon_{2}=\epsilon+\lambda h(a)}}\left\{\partial_{\epsilon_{1}} \lambda h(a)+N_{\epsilon_{2}}(C, a)\right\} .
$$

Here $(\lambda h)^{*} \oplus \delta_{C}^{*}$ denotes the infimum convolution defined, for any $x^{*}$ in $X^{*}$ by:

$$
\left[(\lambda h)^{*} \oplus \delta_{C}^{*}\right]\left(x^{*}\right):=\min _{u \in X^{*}}\left[(\lambda h)^{*}(u)+\delta_{C}^{*}\left(x^{*}-u\right)\right] .
$$

Proof. Let us consider $f \equiv 0$. Then, it comes that $f^{*}(0)=0, \operatorname{dom} f^{*}=\{0\}$ and that

$$
\text { epi } f^{*}+K=\{0\} \times[0,+\infty)+K=K .
$$

Since for each $\epsilon \geq 0, \partial_{\epsilon} \delta_{A}(a)=N_{\epsilon}(A, a)$, the conclusion follows from Theorem 3.1. 


\section{Toland-Fenchel-Lagrange duality for DC programs with convex constraints}

Duality results are useful in the study of the primal problems. In particular, for DC programs, they have been used successfully in building numerical methods for the primal problems (see, for instance, [3], [4] and references therein).

In this section, we are interested in a dual problem for the DC program (P) called "TolandFenchel-Lagrange" dual problem. This type of dual problem was considered in [28] for problems of model (P) and in [29] for DC programs with a finite DC constraints. It is, in some sense, a "combination" of Toland dual problem (for DC problem) in [32], Fenchel and Lagrange dual problems (see $[8,9,23]$ ). We propose several duality results of this type for $(\mathrm{P})$ which extend Laghdir's one in [28]. As consequences of these results, we obtained various corresponding results for convex programs which go back, and in some cases extend, the Fenchel-Lagrange duality or Lagrange duality results in [8], [9], [23], and [17]. So, "Toland-Fenchel-Lagrange" duality serves as a generalization of these types of dual problems to DC programs.

\subsection{Toland-Fenchel-Lagrange duality for DC programs}

In this section, we are interested in the duality results for the $\mathrm{DC}$ problem $(\mathrm{P})$ given by:

$$
\begin{aligned}
\inf & (f(x)-g(x)) \\
\text { subject to } & x \in C, h(x) \in-S .
\end{aligned}
$$

Let us consider this first result. Then, other forms of duality would be derived from this one.

Theorem 4.1 (Toland-Fenchel-Lagrange duality) Suppose $(C C)$ holds, then

$$
\inf (P)=\inf _{x^{*} \in X^{*}} \max _{\lambda \in S^{+}}\left\{g^{*}\left(x^{*}\right)-\left(f+\lambda h+\delta_{C}\right)^{*}\left(x^{*}\right)\right\} .
$$

Proof. Thanks to the Toland dual theorem recalled in Lemma 2.1,

$$
\inf (P)=\inf _{x \in X}\left(f+\delta_{A}-g\right)(x)=\inf _{x^{*} \in X^{*}}\left\{g^{*}\left(x^{*}\right)-\left(f+\delta_{A}\right)^{*}\left(x^{*}\right)\right\} .
$$

Since (CC) holds, it follows from Corollary 3.1 that

$$
\left(f+\delta_{A}\right)^{*}\left(x^{*}\right)=\min _{\lambda \in S^{+}}\left(f+\lambda h+\delta_{C}\right)^{*}\left(x^{*}\right) .
$$

This equality and (16) lead to

$$
\begin{aligned}
\inf (P) & =\inf _{x^{*} \in X^{*}}\left\{g^{*}\left(x^{*}\right)-\left(f+\delta_{A}\right)^{*}\left(x^{*}\right)\right\} . \\
& =\inf _{x^{*} \in X^{*}} \max _{\lambda \in S^{+}}\left\{g^{*}\left(x^{*}\right)-\left(f+\lambda h+\delta_{C}\right)^{*}\left(x^{*}\right)\right\},
\end{aligned}
$$

which completes the proof.

Theorem 4.1 was proved in [29]-Corollary 4.1 (p. 666) where $C=X, h=\left(g_{1}, g_{2}, \ldots, g_{m}\right)$ and $g_{i}$, $i=1,2, \ldots, m$ are extended real-valued convex functions and under Slater constraints qualification. Other forms of strong Toland-Fenchel-Lagrange duality are given in the following theorem.

Theorem 4.2 (Toland-Fenchel-Lagrange duality) Suppose that the condition (CC) holds, then

$$
\begin{aligned}
\inf (P) & =\inf _{x^{*} \in X^{*}}\left\{\max _{\lambda \in S^{+}} \max _{u, v \in X^{*}}\left\{g^{*}\left(x^{*}\right)-f^{*}(u)-(\lambda h)^{*}(v)-\delta_{C}^{*}\left(x^{*}-u-v\right)\right\},\right. \\
\inf (P) & =\inf _{x^{*} \in X^{*}}\left\{\max _{\lambda \in S^{+}} \max _{u \in X^{*}}\left\{g^{*}\left(x^{*}\right)-f^{*}(u)-\left(\lambda h+\delta_{C}\right)^{*}\left(x^{*}-u\right)\right\}\right\}, \\
\inf (P) & =\inf _{x^{*} \in X^{*}}\left\{\max _{\lambda \in S^{+}} \max _{u \in X^{*}}\left\{g^{*}\left(x^{*}\right)-\left(f+\delta_{C}\right)^{*}(u)-(\lambda h)^{*}\left(x^{*}-u\right)\right\}\right\} .
\end{aligned}
$$


Proof. Since (CC) holds, by Theorem 3.1, for each $x^{*} \in X^{*}$,

$$
\left(f+\delta_{A}\right)^{*}\left(x^{*}\right)=\min _{\lambda \in S^{+}} \min _{u, v \in X^{*}}\left(f^{*}(u)+(\lambda h)^{*}(v)+\delta_{C}^{*}\left(x^{*}-u-v\right)\right) .
$$

Combining (16) and (20), we get that

$$
\begin{aligned}
\inf (P) & =\inf _{x^{*} \in X^{*}}\left\{g^{*}\left(x^{*}\right)-\left(f+\delta_{A}\right)^{*}\left(x^{*}\right)\right\} \\
& =\inf _{x^{*} \in X^{*}}\left\{g^{*}\left(x^{*}\right)-\min _{\lambda \in S^{+}} \min _{u, v \in X^{*}}\left(f^{*}(u)+(\lambda h)^{*}(v)+\delta_{C}^{*}\left(x^{*}-u-v\right)\right)\right\} \\
& =\inf _{x^{*} \in X^{*}}\left\{\max _{\lambda \in S^{+}} \max _{u, v \in X^{*}}\left\{g^{*}\left(x^{*}\right)-f^{*}(u)-(\lambda h)^{*}(v)-\delta_{C}^{*}\left(x^{*}-u-v\right)\right\}\right\} .
\end{aligned}
$$

The equality (17) has been proved and similarly, we get (18) and (19)

When the set constraint is absent (i.e., $C=X$ ), we get

Corollary 4.1 Assume that $C=X$ and that the condition $(C C)$ holds, then

$$
\inf (P)=\inf _{x^{*} \in X^{*}}\left\{\max _{\lambda \in S^{+}} \max _{u \in X^{*}}\left\{g^{*}\left(x^{*}\right)-f^{*}(u)-(\lambda h)^{*}\left(x^{*}-u\right)\right\}\right\} .
$$

Proof. The proof is similar to Theorem 4.2 one, by using Corollary 3.2 instead of Theorem 3.1.

Example 4.1. Consider the following problem (E1):

$$
\begin{array}{cl}
\inf & \left(x_{1}^{2}-x_{2}-\sqrt{x_{1}^{2}+x_{2}^{2}}\right) \\
\text { subject to } & x=\left(x_{1}, x_{2}\right) \in \mathbb{R}^{2}, x_{1}+x_{2} \leq 0 .
\end{array}
$$

Let $f(x)=x_{1}^{2}-x_{2}, g(x)=\sqrt{x_{1}^{2}+x_{2}^{2}}, X=\mathbb{R}^{2}, C=X, Z=\mathbb{R}, S=S^{+}=\mathbb{R}_{+}, h(x)=x_{1}+x_{2}$. It is clear that $f, g$ are convex, continuous functions on $\mathbb{R}^{2}$, while $h$ is $S$-convex and continuous. Then (E1) has the form of $(\mathrm{P})$.

For each $u=\left(u_{1}, u_{2}\right) \in \mathbb{R}^{2}$, one has that

$$
f^{*}(u)=\sup _{x \in \mathbb{R}^{2}}\left(u_{1} x_{1}+u_{2} x_{2}-x_{1}^{2}+x_{2}\right)= \begin{cases}\frac{u_{1}^{2}}{4} & \text { if } u_{2}=-1 \\ +\infty & \text { if } u_{2} \neq-1 .\end{cases}
$$

For each $v=\left(v_{1}, v_{2}\right) \in \mathbb{R}^{2}$ and $\lambda \in S^{+}=\mathbb{R}_{+}$, it comes that

$$
(\lambda h)^{*}(v)=\sup _{x \in \mathbb{R}^{2}}\left(v_{1} x_{1}+v_{2} x_{2}-\lambda x_{1}-\lambda x_{2}\right)= \begin{cases}0 & \text { if } v_{1}=v_{2}=\lambda, \\ +\infty & \text { otherwise. }\end{cases}
$$

For each $a=\left(a_{1}, a_{2}\right) \in \mathbb{R}^{2}$, one gets that

$$
\begin{aligned}
g^{*}(a) & =\sup _{x \in \mathbb{R}^{2}}\left(a_{1} x_{1}+a_{2} x_{2}-\sqrt{x_{1}^{2}+x_{2}^{2}}\right) \\
& \leq \sup _{x \in \mathbb{R}^{2}}\left(\sqrt{a_{1}^{2}+a_{2}^{2}}-1\right) \sqrt{x_{1}^{2}+x_{2}^{2}} .
\end{aligned}
$$

(the last inequality follows from the Cauchy-Bunhiakovski's inequality). This implies that

$$
g^{*}(a)= \begin{cases}0 & \text { if } a_{1}^{2}+a_{2}^{2} \leq 1 \\ +\infty & \text { if } a_{1}^{2}+a_{2}^{2}>1\end{cases}
$$

Therefore,

$$
\text { epi } f^{*}+\cup_{\lambda \in S^{+}} \text {epi } h^{*}=\left\{\left(u_{1}+\lambda, \lambda-1, \frac{u_{1}^{2}}{4}+r\right) \mid u_{1} \in \mathbb{R},(\lambda, r) \in \mathbb{R}_{+}^{2}\right\}
$$


which is a closed subset of $\mathbb{R}^{3}$. By Corollary 4.1 , we get that

$$
\inf (E 1)=\inf _{\substack{a_{1}^{2}+a_{2}^{2} \leq 1 \\\left(a_{1}, a_{2}\right) \in \mathbb{R}^{2}}} \max _{\substack{a_{2}-\lambda=-1 \\ \lambda \geq 0}}-\frac{\left(a_{1}-\lambda\right)^{2}}{4}=\inf _{\substack{a_{1}^{2}+a_{2}^{2} \leq 1 \\\left(a_{1}, a_{2}\right) \in \mathbb{R}^{2}}}-\frac{\left(a_{1}-a_{2}-1\right)^{2}}{4} .
$$

Note that

$$
\left|a_{1}-a_{2}-1\right| \leq\left|a_{1}-a_{2}\right|+1 \leq \sqrt{2} \sqrt{a_{1}^{2}+a_{2}^{2}}+1 .
$$

Therefore, $\inf (E 1)=-\frac{(\sqrt{2}+1)^{2}}{4}$ and the infimum in the right hand side of the last equality is attained at $a_{1}=\frac{-1}{\sqrt{2}}, a_{2}=\frac{1}{\sqrt{2}}$.

It is worth observing that the conclusions of Theorems 4.1 and 4.2-(17) were established in [28] (Propositions 4.1, 4.2, 4.3 and Corollary 4.1) with the assumptions that $f$ is continuous at one point in $C$ and either

$(\alpha) \bar{x} \in C$ exists such that $h(\bar{x}) \in-\operatorname{int} S$ (Slater constraint qualification),

or

$(\beta) \mathbb{R}_{+}[S+h(\operatorname{dom} f \cap C)]$ is a closed subspace.

Let us remind (see [23]) that if the Slater condition $(\alpha)$ holds, then $\sigma$ is (FM). Thus, with the fact that $f$ is continuous at one point in $C$, it comes that (CC) holds.

Let us see in the next corollary that the same conclusion holds with the assumption $(\beta)$.

Corollary 4.2 If $f$ is continuous at some point in $C$ and $\mathbb{R}_{+}[S+h(\operatorname{domf} \cap \mathrm{C})]$ is a closed subspace of $Z$ then $(C C)$ holds.

Proof. Assume that the hypothesis of the corollary holds. Let $x_{0}^{*} \in X^{*}$ be arbitrary and set $g=x_{0}^{*}$. Since $g^{*}\left(x_{0}^{*}\right)=0$ if $x^{*}=x_{0}^{*}$ and $g^{*}\left(x^{*}\right)=+\infty$ else, it comes from [28]-Corollary 4.1, that

$$
\begin{aligned}
\inf (P) & =\inf _{x^{*} \in X^{*}}\left\{\max _{\lambda \in S^{+}} \max _{u, v \in X^{*}}\left\{g^{*}\left(x^{*}\right)-f^{*}(u)-(\lambda h)^{*}(v)-\delta_{C}^{*}\left(x^{*}-u-v\right)\right\}\right\} \\
& =\max _{\lambda \in S^{+}} \max _{u, v \in X^{*}}\left\{-f^{*}(u)-(\lambda h)^{*}(v)-\delta_{C}^{*}\left(x_{0}^{*}-u-v\right)\right\} .
\end{aligned}
$$

Observe that $\inf (P)=\inf _{x \in A}\left\{f(x)-\left(x_{0}^{*}, x\right)\right\}=-\left(f+\delta_{A}\right)^{*}\left(x_{0}^{*}\right)$. Therefore,

$$
\left(f+\delta_{A}\right)^{*}\left(x_{0}^{*}\right)=\min _{\lambda \in S^{+}} \min _{u, v \in X^{*}}\left\{f^{*}(u)+(\lambda h)^{*}(v)+\delta_{C}^{*}\left(x_{0}^{*}-u-v\right)\right\} .
$$

Since the last equality holds for all $x_{0}^{*} \in X^{*},(\mathrm{CC})$ follows from Theorem 3.1.

Corollary 4.2 gives a sufficient condition to (CC). It is not a necessary one, as the following simple example shows.

Example 4.2. Consider the simple case when $X=\mathbb{R}, C=[-1,1], S=\mathbb{R}_{+}, f(x):=x, g(x) \equiv 0$, and $h(x)=\max \{0, x\}$. Then, for $a \in \mathbb{R}$ and $\lambda \in \mathbb{R}_{+}$, we have that $\left(\delta_{C}\right)^{*}(a)=|a|$ and that

$$
f^{*}(a)=\left\{\begin{array}{ll}
0 & \text { if } a=1, \\
+\infty & \text { if } a \neq 1,
\end{array} \quad(\lambda h)^{*}(a)= \begin{cases}0 & \text { if } a \in[0, \lambda] \\
+\infty & \text { if } a \notin[0, \lambda]\end{cases}\right.
$$

Then, epif* $f^{*} \bigcup_{\lambda \in \mathbb{R}_{+}}(\lambda h)^{*}+\operatorname{epi} \delta_{C}^{*}=\{1\} \times \mathbb{R}_{+}+\mathbb{R}_{+} \times \mathbb{R}_{+}+$epi $|$.$| is a closed subset of \mathbb{R}^{2}$ while $\mathbb{R}_{+}\left[\mathbb{R}_{+}+h(\operatorname{dom} f \cap C)\right]=\mathbb{R}_{+}\left[\mathbb{R}_{+}+h(\mathbb{R})\right]=[0,+\infty)$ is not a closed subspace of $\mathbb{R}$.

\subsection{Duality for convex programs}

Let us derive in this section results about the convex case from the DC one. In order to, consider the convex program:

$$
\begin{aligned}
& \text { (Q) } \quad \inf f(x) \\
& \text { subject to } x \in C, h(x) \in-S \text {. }
\end{aligned}
$$

Then, the problem $(\mathrm{Q})$ is a special case of $(\mathrm{P})$, where $g \equiv 0$. 
Lagrange duality.

Let us recall that the Lagrange duality form such a problem is given by

$$
\text { (LDQ) } \sup _{\lambda \in S^{+}}\left\{\inf _{x \in C}(f+\lambda h)(x)\right\} \text {. }
$$

Then, we find the strong duality for the Lagrange dual problem, as established recently in [16].

Corollary 4.3 (Lagrange duality) Suppose that $(C C)$ holds, then the Lagrange strong duality holds for $(Q)$, that is,

$$
\inf (Q)=\max _{\lambda \in S^{+}}\left\{\inf _{x \in C}(f+\lambda h)(x)\right\}
$$

(the Problem $(L D Q)$ is solvable).

Proof. Note that if $g \equiv 0$ then $g^{*}(0)=0$ and $g^{*}\left(x^{*}\right)=+\infty$ if $x^{*} \in X^{*} \backslash\{0\}$. Since (CC) holds, by Theorem 4.1, applied to the case where $g \equiv 0$, we get

$$
\begin{aligned}
\inf (Q) & =\max _{\lambda \in S^{+}}\left\{-\left(f+\lambda h+\delta_{C}\right)^{*}(0)\right\} \\
& =\max _{\lambda \in S^{+}}\left\{-\sup _{x \in C}\{-f(x)-\lambda h(x)\}\right\} \\
& =\max _{\lambda \in S^{+}}\left\{\inf _{x \in C}(f(x)+\lambda h(x))\right\} .
\end{aligned}
$$

The proof is complete.

Fenchel-Lagrange duality.

The Fenchel-Lagrange dual problem of $(\mathrm{Q})$ is defined by [8]:

(FLDQ) $\max _{\lambda \in S^{+}} \max _{u, v \in X^{*}}\left\{-f^{*}(u)-(\lambda h)^{*}(v)-\delta_{C}^{*}(-u-v)\right\}$.

As a consequence of Theorem 4.2, we get the following strong Fenchel-Lagrange duality result for (Q).

Corollary 4.4 (Fenchel-Lagrange duality) Suppose that $(C C)$ holds, then the strong duality holds between $(Q)$ and $(F L D Q)$, i.e.:

$$
\inf (Q)=\max _{\lambda \in S^{+}} \max _{u, v \in X^{*}}\left\{-f^{*}(u)-(\lambda h)^{*}(v)-\delta_{C}^{*}(-u-v)\right\} .
$$

Proof. Let $g \equiv 0$. Since $g^{*}(0)=0$ and $g^{*}\left(x^{*}\right)=+\infty$ if $x^{*} \in X^{*} \backslash\{0\}$, the results follows from the first assertion of Theorem 4.2.

The Fenchel-Lagrange strong duality result given in Corollary 4.4 was established in [8] (see also [7]) under the assumptions that: $X=\mathbb{R}^{n}, h=\left(h_{1}, h_{2}, \cdots, h_{m}\right), h_{i}: \mathbb{R}^{n} \longrightarrow \mathbb{R}$ convex $\left(S=\mathbb{R}_{+}^{m}\right)$ and under the Slater constraint qualification condition stating that $\operatorname{ri} C \cap \operatorname{ri}(\operatorname{dom} f) \neq \emptyset$. Moreover, it was supposed that $x^{\prime} \in \operatorname{ri} C \cap \operatorname{ri}(\operatorname{dom} f)$ exists such that $h_{i}\left(x^{\prime}\right)<0, i=1,2, \ldots, m$. Again, if $f$ is continuous at some point in $A$, since the condition (CC) holds if (FM) is assumed, our condition is strictly weaker than the Slater's one (see [23]).

Let us present in the following corollary, other forms of Fenchel-Lagrange duality. The first one was established recently in [9] with: $X, Z$ Banach spaces, $f$ a continuous convex function and $g$ a $S$-convex, continuous mapping.

Corollary 4.5 (Fenchel-Lagrange duality) Suppose that $(C C)$ holds, then

$$
\begin{aligned}
& \inf (Q)=\max _{\lambda \in S^{+}} \max _{u \in X^{*}}\left\{-f^{*}(u)-\left(\lambda h+\delta_{C}\right)^{*}(-u)\right\} \\
& \inf (Q)=\max _{\lambda \in S^{+}} \max _{u \in X^{*}}\left\{-\left(f+\delta_{C}\right)^{*}(u)-(\lambda h)^{*}(-u)\right\} .
\end{aligned}
$$

Proof. This is a direct consequence of Theorem 4.2 (see (18) and (19)) by letting $g \equiv 0$. 


\section{Generalized Farkas lemmas}

Let us use the duality results obtained in the previous section to derive generalized Farkas lemmas in dual forms for systems involving convex and DC functions. All the assumptions on the functions $f, g$, the mapping $h$, the spaces $X, Z$, the cone $S$ and the subset $C$ are as in Section 1 .

Theorem 5.1 (Farkas lemma) Suppose that $(C C)$ holds and that $\alpha \in \mathbb{R}$. Then, the following statements are equivalent:

(i) $x \in C, h(x) \in-S \Longrightarrow f(x)-g(x) \geq \alpha$,

(ii) For each $x^{*} \in X^{*}$, there exist $\lambda \in S^{+}, u \in X^{*}$ such that

$$
g^{*}\left(x^{*}\right)-f^{*}(u)-\left(\lambda h+\delta_{C}\right)^{*}\left(x^{*}-u\right) \geq \alpha,
$$

(iii) For each $x^{*} \in X^{*}$, there exist $\lambda \in S^{+}, u \in X^{*}$ such that

$$
g^{*}\left(x^{*}\right)-\left(f+\delta_{C}\right)^{*}(u)-(\lambda h)^{*}\left(x^{*}-u\right) \geq \alpha,
$$

(iv) For each $x^{*} \in X^{*}$, there exist $\lambda \in S^{+}, u, v \in X^{*}$ such that

$$
g^{*}\left(x^{*}\right)-f^{*}(u)-(\lambda h)^{*}(v)-\delta_{C}^{*}\left(x^{*}-u-v\right) \geq \alpha .
$$

Proof. $\quad[(i) \Rightarrow(i v)]$ Suppose that (i) holds, then $\inf (P) \geq \alpha$. Since (CC) holds, Theorem 4.2 leads to

$$
\inf (P)=\inf _{x^{*} \in X^{*}} \max _{\lambda \in S^{+}} \max _{u, v \in X^{*}}\left\{g^{*}\left(x^{*}\right)-f^{*}(u)-(\lambda h)^{*}(v)-\delta_{C}^{*}\left(x^{*}-u-v\right)\right\} .
$$

Then, for each $x^{*} \in X^{*}$, there exist $\lambda \in S^{+}$and $u, v \in X^{*}$ such that

$$
g^{*}\left(x^{*}\right)-f^{*}(u)-(\lambda h)^{*}(v)-\delta_{C}^{*}\left(x^{*}-u-v\right) \geq \inf (P) \geq \alpha,
$$

which proves (iv).

$[(i v) \Rightarrow(i)]$ Suppose that (iv) holds. Then, for each $x^{*} \in X^{*}$, there exist $\lambda \in S^{+}$and $u \in X^{*}$, such that $g^{*}\left(x^{*}\right)-f^{*}(u)-(\lambda h)^{*}(v)-\delta_{C}^{*}\left(x^{*}-u-v\right) \geq \alpha$. This implies that

$$
\max _{\lambda \in S^{+}} \max _{u \in X^{*}}\left(g^{*}\left(x^{*}\right)-f^{*}(u)-(\lambda h)^{*}(v)-\delta_{C}^{*}\left(x^{*}-u-v\right)\right) \geq \alpha .
$$

Therefore, it comes that

$$
\inf _{x^{*} \in X^{*}} \max _{\lambda \in S^{+}} \max _{u, v \in X^{*}}\left(g^{*}\left(x^{*}\right)-f^{*}(u)-(\lambda h)^{*}(v)-\delta_{C}^{*}\left(x^{*}-u-v\right)\right) \geq \alpha .
$$

Since (CC) holds, the strong duality holds (Theorem 4.2), we get that $\inf (P) \geq \alpha$, which means that (i) holds.

The equivalence between (i), (ii), and (iii) follows from the same argument as in the proof of the equivalence between (i) and (iv), using (18) or (19) instead of (23).

Let us have a look at the convex systems. As a consequence of Corollaries 4.4 and 4.5 , we get the following generalized versions of Farkas lemma.

Corollary 5.1 (Farkas lemma for convex systems) Suppose that $(C C)$ holds and $\alpha \in \mathbb{R}$. Then, the following statements are equivalent:

(i) $x \in C, h(x) \in-S \Longrightarrow f(x) \geq \alpha$,

(ii) There exist $\lambda \in S^{+}, u \in X^{*}$ such that

$$
-f^{*}(u)-\left(\lambda h+\delta_{C}\right)^{*}(-u) \geq \alpha,
$$

(iii) There exist $\lambda \in S^{+}, u \in X^{*}$ such that

$$
-\left(f+\delta_{C}\right)^{*}(u)-(\lambda h)^{*}(-u) \geq \alpha,
$$

(iv) There exist $\lambda \in S^{+}, u, v \in X^{*}$ such that

$$
-f^{*}(u)-(\lambda h)^{*}(v)-\delta_{C}^{*}(-u-v) \geq \alpha .
$$


Proof. The proof is quite similar to that of Theorem 5.1. Here, Corollaries 4.4 and 4.5 are used instead of Theorem 4.2.

Let us mention that the equivalence between (i) and (ii) in Corollary 5.1 was proved in [8]-Theorem 4.1 (p. 545) under the assumptions: $X=\mathbb{R}^{n}, \alpha=0, h=\left(h_{1}, h_{2}, \cdots, h_{m}\right)$ where $h_{i}: \mathbb{R}^{n} \longrightarrow \mathbb{R}$ convex $\left(S=\mathbb{R}_{+}^{n}\right)$ and under the Slater constraint qualification condition. In [8], a convex problem with $X=\mathbb{R}^{n}, g_{i}(x) \leq 0$ for all $i \in I$ (possibly infinite set) was considered as well. A version of Farkas lemma was proved. It is similar to the equivalence of (i) and (ii) in Corollary 5.1, but without showing explicitly the existence of " $\lambda \in S^{+}$" (Theorem 5.2, page 549). Note that such a problem can be reduced to the model (Q) by letting $h: \mathbb{R}^{n} \rightarrow \mathbb{R}^{I}$ with $h(x)=\left(h_{i}(x)\right)_{i \in I}$ and $S=\mathbb{R}_{+}^{I}$ (see $[15,16])$. For more versions of Farkas lemma involving convex and DC functions, see [18].

\section{Optimality condition for DC programs with convex con- straints}

In this section, we will establish necessary and sufficient conditions for global optimality for the problem $(\mathrm{P})$.

Theorem 6.1 Let $\bar{x} \in A \cap \operatorname{dom} f$ and assume that the condition $(C C)$ holds. Then, $\bar{x}$ is a global solution of $(P)$ if and only if for each $\epsilon \geq 0$ and each $x^{*} \in \partial_{\epsilon} g(\bar{x})$, there exist $\lambda \in S^{+}$and $\epsilon_{1}, \epsilon_{2}, \epsilon_{3} \geq 0$ such that $\epsilon_{1}+\epsilon_{2}+\epsilon_{3}=\epsilon+\lambda h(\bar{x})$ and

$$
x^{*} \in \partial_{\epsilon_{1}} f(\bar{x})+\partial_{\epsilon_{2}}(\lambda h)(\bar{x})+N_{\epsilon_{3}}(C, \bar{x}) .
$$

In particular, if $\bar{x} \in A$ is a solution of $(P)$, then, for each $x^{*} \in \partial g(\bar{x}), \lambda \in S^{+}$exists such that $x^{*} \in \partial f(\bar{x})+\partial(\lambda h)(\bar{x})+N(C, \bar{x})$ and $\lambda h(\bar{x})=0$.

Proof. It is clear that Problem $(\mathrm{P})$ can be written in the form:

$$
\left(\mathrm{P}^{\prime}\right) \quad \inf _{x \in X}\left[\left(f+\delta_{A}\right)(x)-g(x)\right] .
$$

By Lemma 2.2, $\bar{x}$ is a global solution of $(\mathrm{P})$ if and only if for each $\epsilon \geq 0$,

$$
\partial_{\epsilon} g(\bar{x}) \subset \partial_{\epsilon}\left(f+\delta_{A}\right)(\bar{x}) .
$$

Since (CC) holds, it follows from Theorem 3.1 that

$$
\partial_{\epsilon}\left(f+\delta_{A}\right)(\bar{x})=\bigcup_{\lambda \in S^{+}} \bigcup_{\substack{\epsilon_{1}, \epsilon_{2}, \epsilon_{3} \geq 0 \\ \epsilon_{1}+\epsilon_{2}+\epsilon_{3}=\epsilon+\lambda h(\bar{x})}}\left\{\partial_{\epsilon_{1}} f(\bar{x})+\partial_{\epsilon_{2}} \lambda h(\bar{x})+N_{\epsilon_{3}}(C, \bar{x})\right\} .
$$

Thus, $\bar{x}$ is a global solution of $(\mathrm{P})$ if and only if for each $\epsilon \geq 0$,

$$
\partial_{\epsilon} g(\bar{x}) \subset \bigcup_{\lambda \in S^{+}} \bigcup_{\substack{\epsilon_{1}, \epsilon_{2}, \epsilon_{3} \geq 0 \\ \epsilon_{1}+\epsilon_{2}+\epsilon_{3}=\epsilon+\lambda h(\bar{x})}}\left\{\partial_{\epsilon_{1}} f(\bar{x})+\partial_{\epsilon_{2}} \lambda h(\bar{x})+N_{\epsilon_{3}}(C, \bar{x})\right\}
$$

which means that for each $x^{*} \in \partial_{\epsilon} g(\bar{x})$, there exists $\lambda \in S^{+}$and $\epsilon_{1}, \epsilon_{2}, \epsilon_{3} \geq 0$ such that $\epsilon_{1}+\epsilon_{2}+\epsilon_{3}=$ $\epsilon+\lambda h(\bar{x})$ and $x^{*} \in \partial_{\epsilon_{1}} f(\bar{x})+\partial_{\epsilon_{2}}(\lambda h)(\bar{x})+N_{\epsilon_{3}}(C, \bar{x})$. The first assertion is proved.

Now, if $\bar{x} \in A$ is a solution of (P), then $\lambda h(\bar{x}) \leq 0$ (as $\lambda \in S^{+}$and $\bar{x} \in A$ ). So, if $\lambda \in S^{+}$and $\epsilon_{1}, \epsilon_{2}, \epsilon_{3} \geq 0$ satisfying $\epsilon_{1}+\epsilon_{2}+\epsilon_{3}=\lambda h(\bar{x})$, then $\epsilon_{1}=\epsilon_{2}=\epsilon_{3}=\lambda h(\bar{x})=0$. The last assertion follows from the previous observation and the first assertion of the theorem with $\epsilon=0$.

Example 6.1. Consider the problem

$$
\begin{aligned}
\inf & {\left[\left(x^{4}+x\right)-x^{2}\right] } \\
\text { subject to } & x \in[-1 ; 1], \max (0,-x) \leq 0 .
\end{aligned}
$$


Let $f(x)=x^{4}+x, g(x)=x^{2}, h(x)=\max (0,-x), X=X^{*}=\mathbb{R}, C=[-1 ; 1], Z=Z^{*}=\mathbb{R}$ and $S=S^{+}=\mathbb{R}_{+}$. It is obvious that $f, g$ are continuous convex functions and that $h$ is continuous and $S$-convex. The Problem (E2) has the form of (P).

It is easy to see that for $\lambda \in S^{+}=\mathbb{R}_{+}$and $a \in \mathbb{R}$, we have that

$$
(\lambda h)^{*}(a)=\sup _{x \in X}[(a, x)-(\lambda h)(x)]= \begin{cases}0 & \text { if } a \in[-\lambda, 0] \\ +\infty & \text { otherwise. }\end{cases}
$$

Thus epi $(\lambda h)^{*}=[-\lambda ; 0] \times \mathbb{R}_{+}$and hence, $\cup_{\lambda \in S^{+}}$epi $(\lambda h)^{*}=\mathbb{R}_{-} \times \mathbb{R}_{+}$.

On the other hand, it is clear that epi $\delta_{C}^{*}=$ epi $|$.$| . We have that$

$$
\cup_{\lambda \in S^{+}} \text {epi }(\lambda h)^{*}+\text { epi } \delta_{C}^{*}=\{(x, y) \mid x \leq 0, y \geq 0\} \cup\{(x, y) \mid y \geq x \geq 0\}
$$

is a closed subset of $\mathbb{R}^{2}$. Since $f$ is continuous on $\mathbb{R}$, epi $f^{*}+\cup_{\lambda \in S^{+}}$epi $(\lambda h)^{*}+$ epi $\delta_{C}^{*}$ is closed in $\mathbb{R}^{2}$, which means that (CC) holds for the Problem (E2).

Let us consider $\epsilon, \epsilon_{1}, \epsilon_{2}, \epsilon_{3} \geq 0$ and $\lambda \in \mathbb{R}_{+}$then, we have that

$$
\begin{array}{ll}
\partial_{\epsilon} g(0)=[-2 \sqrt{\epsilon}, 2 \sqrt{\epsilon}], & \partial_{\epsilon_{1}} f(0)=\left[1-4 \sqrt[4]{\frac{\epsilon_{1}^{3}}{27}}, 1+4 \sqrt[4]{\frac{\epsilon_{1}^{3}}{27}}\right], \\
\forall \lambda \in S^{+}, \partial_{\epsilon_{2}} \lambda h(0)=[-\lambda, 0] \quad \text { and } \quad N_{\epsilon_{3}}(C, 0)=\left[-\epsilon_{3}, \epsilon_{3}\right]
\end{array}
$$

Choose $\epsilon_{1}=\epsilon_{2}=0, \epsilon_{3}=\epsilon$ and $\lambda \geq 0$ satisfying $-2 \sqrt{\epsilon} \geq 1-\lambda-\epsilon$. Since $\epsilon+1 \geq 2 \sqrt{\epsilon}$, we have that

$$
\begin{aligned}
\partial_{\epsilon} g(0) & =[-2 \sqrt{\epsilon},-2 \sqrt{\epsilon}] \\
& \subset[1-\lambda-\epsilon, 1+\epsilon]=\{1\}+[-\lambda, 0]+[-\epsilon, \epsilon] \\
& =\partial_{\epsilon_{1}} f(0)+\partial_{\epsilon_{2}}\left(\lambda h(0)+N_{\epsilon_{3}}(C, 0)\right.
\end{aligned}
$$

By Theorem $6.1, \bar{x}=0$ is a global solution of (E3).

By taking $g \equiv 0$ in Theorem 6.1 , we will get an optimality condition for the convex Problem (Q) considered in Section 4.

Corollary 6.1 Suppose that (CC) holds and that $\bar{x} \in A$. Then, $\bar{x}$ is a (global) solution of (Q) if and only if there exists $\lambda \in S^{+}$such that

$$
0 \in \partial f(\bar{x})+\partial(\lambda h)(\bar{x})+N(C, \bar{x}), \quad \lambda h(\bar{x})=0 .
$$

Proof. Necessity. Suppose that $\bar{x}$ is an optimal solution of (Q). Theorem 6.1 with $g \equiv 0$ and with $\epsilon=0$ ensures the existence of $\lambda \in S^{+}$, such that (25) holds. Then, the condition $\lambda h(\bar{x})=0$ follows from an argument similar to the last part of the proof of Theorem 6.1.

Sufficiency. Suppose that there exists $\lambda \in S^{+}$such that (25) holds. Then we can find $u \in \partial f(\bar{x})$, $v \in \partial(\lambda h)(\bar{x})$ and $w \in N(C, \bar{x})$ such that $u+v+w=0$. By definition of subdifferentials of convex functions,

$$
\begin{aligned}
f(x)-f(\bar{x}) & \geq(u, x-\bar{x}) \forall x \in X, \\
\lambda h(x)-\lambda h(\bar{x}) & \geq(v, x-\bar{x}) \forall x \in X, \\
0 & \geq(w, x-\bar{x}) \quad \forall x \in C .
\end{aligned}
$$

Since $\lambda h(\bar{x})=0$, these inequalities imply that $f(x)+\lambda h(x)-f(\bar{x}) \geq 0$, for all $x \in C$. Note that for all $x \in A, \lambda h(x) \leq 0$. Combining these facts, we get $f(x) \geq f(\bar{x})$ for all $x \in A$, which means that $\bar{x}$ is a solution of $(\mathrm{Q})$.

The optimality condition given in Corollary 6.1 was established in [27]. It was also established in $[11,23,24]$ by assuming that $X$ was a Banach space, $\sigma$ was (FM) and either $f$ was continuous, or $C=X$, or epi $f^{*}+\mathrm{cl} K$ was weak*-closed. In [14], this optimality condition was also proved under the assumption that $f$ was continuous at one point in $C, h$ was sequentially l.s.c. and $\mathbb{R}_{+}[S+h(C)]$ was a closed subspace. In either of the cases, (CC) holds. Similar assumptions were imposed in [16], when dealing with the convex infinite problems to get the same kind of optimality condition for this class of problems. 
Let us consider now the problem of maximizing a convex function under convex constraints.

$$
\begin{array}{lll}
\text { sup } & p(x) \\
\text { subject to } & x \in C, h(x) \in-S,
\end{array}
$$

where $X, C, S, h$ are as in the previous sections and $p: X \rightarrow \mathbb{R} \cup\{+\infty\}$ is a proper, l.s.c. and convex function.

It is clear that if $\bar{x} \in A$ is a (global) solution of the problem (CMP), then it is a solution of the problem (CMP1) below.

$$
\begin{aligned}
& \text { (CMP1) inf }-p(x) \\
& \text { subject to } x \in C, h(x) \in-S \text {. }
\end{aligned}
$$

As a direct consequence of Theorem 6.1, we get:

Corollary 6.2 Suppose that $\sigma$ is (FM) and that $\bar{x} \in A$. Then, $\bar{x}$ is a global solution of (CMP) if and only if for each $\epsilon \geq 0$ and each $x^{*} \in \partial_{\epsilon} p(\bar{x})$, there exist $\lambda \in S^{+}$and $\epsilon_{1}, \epsilon_{2} \geq 0$ such that $\epsilon_{1}+\epsilon_{2}=\epsilon+\lambda h(\bar{x})$ and

$$
x^{*} \in \partial_{\epsilon_{1}}(\lambda h)(\bar{x})+N_{\epsilon_{2}}(C, \bar{x}) .
$$

In particular, if $\bar{x} \in A$ is a solution $(C M P)$, then for each $x^{*} \in \partial g(\bar{x})$, there exists $\lambda \in S^{+}$such that $x^{*} \in \partial(\lambda h)(\bar{x})+N(C, \bar{x})$ and $\lambda h(\bar{x})=0$.

\section{An application: DC programs with semi-definite constraints}

In this section, as an application of the results presented in the previous sections, we derive some results on the duality and optimality conditions for a class of DC programs with semi-definite constraints. When $g \equiv 0$, it reduces to a convex program with semi-definite constraints. Many real problems lead to mathematical models such as, finding the distance between two polyhedra, or convex quadratic optimization problems with semi-definite constraints (see [6] and [10]). In this case, the results cover some known results proved recently in [24, 26].

Consider the DC program with a semi-definite constraint:

$$
\begin{array}{lll}
(\mathrm{SDP}) & \inf & {[f(x)-g(x)]} \\
\text { subject to } & x \in C, F_{0}+\sum_{i=1}^{m} x_{i} F_{i} \succeq 0
\end{array}
$$

where $C$ is a closed convex subset of $X=\mathbb{R}^{m}, f, g: \mathbb{R}^{m} \rightarrow \mathbb{R}$ are convex functions, $F_{i} \in S_{n}$, where $S_{n}$ is the space of symmetric $(n \times n)$-matrices. Here $\succeq$ denotes the Löwer partial order of $S_{n}$, that is, for $M, N \in S_{n}, M \succeq N$ means that $M-N$ is a positive semi-definite matrix. $S_{n}$ will be considered as a vector space with the trace inner product defined by $(M, N):=\operatorname{Tr}[M N]$, where $\operatorname{Tr}[$.$] is the$ trace operation. Let $S=\left\{M \in S_{n} \mid M \succeq 0\right\}$ be the closed convex cone of positive semi-definite $(n \times n)$-matrices. Then $S^{+}=S$ and $M \in S$ if and only if $\operatorname{Tr}[Z M] \geq 0$ for all $Z \in S$. The feasible set of (SDP) is $A=\left\{x \in X \mid x \in C, F_{0}+\sum_{i=1}^{m} x_{i} F_{i} \succeq 0\right\}$. For each $x \in \mathbb{R}^{m}$ and $Z \in S_{n}$, let us denote by $F(x)=F_{0}+\sum_{i=1}^{m} x_{i} F_{i}, \hat{F}(x)=\sum_{i=1}^{m} x_{i} F_{i}$ and $\hat{F}^{*}(Z)=\left(\operatorname{Tr}\left[F_{1} Z\right], \ldots, \operatorname{Tr}\left[F_{m} Z\right]\right)$.

Then the Problem (SDP) can be rewritten in the form of $(\mathrm{P})$ as follows:

$$
\begin{array}{lll}
(\mathrm{SDP} 1) & \inf & {[f(x)-g(x)]} \\
\text { subject to } & x \in C,-F(x) \in-S .
\end{array}
$$

Note that for each $Z \in S$ and $u \in \mathbb{R}^{m}$, we have that

$$
\begin{aligned}
(-Z F)^{*}(u) & =\sup _{x \in \mathbb{R}^{m}}\{(u, x)+(Z, F(x))\} \\
& =\sup _{x \in \mathbb{R}^{m}}\left\{(u, x)+\sum_{i=1}^{m} x_{i} \operatorname{Tr}\left[Z F_{i}\right]+\operatorname{Tr}\left[Z F_{0}\right]\right\} \\
& =\operatorname{Tr}\left[Z F_{0}\right]+\sup _{x \in \mathbb{R}^{m}}\left(u+\hat{F}^{*}(Z), x\right) .
\end{aligned}
$$


Therefore,

$$
(-Z F)^{*}(u)= \begin{cases}\operatorname{Tr}\left[Z F_{0}\right] & \text { if } u=-\hat{F}^{*}(Z) \\ +\infty & \text { if } u \neq-\hat{F}^{*}(Z) .\end{cases}
$$

Consequently, for all $Z \in S$, one gets that epi $(-Z F)^{*}=\left(-\hat{F}^{*}(Z), \operatorname{Tr}\left[Z F_{0}\right]\right)+\{0\} \times \mathbb{R}_{+}$and the set $K$ defined by (1) corresponding to the system $\sigma^{*}:=\{x \in C,-F(x) \in-S\}$ becomes

$$
\bigcup_{Z \in S, r \geq 0}\left(-\hat{F}^{*}(Z), \operatorname{Tr}\left[Z F_{0}\right]+r\right)+\operatorname{epi} \delta_{C}^{*} .
$$

So, the system $\sigma^{*}$ is (FM) if and only if this set is closed. Note also that when $C=X$, the condition $(\mathrm{FM})$ for $\sigma^{*}$ reads as: the set $\bigcup_{Z \in S, r \geq 0}\left(-\hat{F}^{*}(Z), \operatorname{Tr}\left[Z F_{0}\right]+r\right)$ is closed in $\mathbb{R}^{m+1}$. This condition (where $C=X$ ) was introduced in [30] and then was used in [24]. The corresponding condition with the presence of the closed convex set $C$, was introduced in [26]. As a consequence of Theorems 4.1 and 4.2 , we get

Theorem 7.1 Assume that $\sigma^{*}$ is (FM), then the following assertions holds.

$$
\begin{aligned}
\inf (S D P) & =\inf _{x^{*} \in \mathbb{R}^{m}}\left\{\max _{Z \in S} \max _{u \in \mathbb{R}^{m}}\left\{g^{*}\left(x^{*}\right)-f^{*}(u)-\delta_{C}^{*}\left(x^{*}-u+\hat{F}^{*}(Z)\right)-\operatorname{Tr}\left[Z F_{0}\right]\right\}\right\}, \\
\inf (S D P) & =\inf _{x^{*} \in \mathbb{R}^{m}}\left\{\max _{Z \in S}\left\{g^{*}\left(x^{*}\right)-\left(f+\delta_{C}\right)^{*}\left(x^{*}+\hat{F}^{*}(Z)\right)-\operatorname{Tr}\left[Z F_{0}\right]\right\}\right\} .
\end{aligned}
$$

Proof. Since $\sigma^{*}$ is (FM) and as $f$ is continuous, (CC) holds and Theorem 4.1 leads to

$$
\begin{aligned}
\inf (S D P 1) & =\inf _{x^{*} \in X^{*}}\left\{\max _{Z \in S^{+}} \max _{u, v \in X^{*}}\left\{g^{*}\left(x^{*}\right)-f^{*}(u)-(-Z F)^{*}(v)-\delta_{C}^{*}\left(x^{*}-u-v\right)\right\}\right\} \\
& =\inf _{x^{*} \in \mathbb{R}^{m}}\left\{\max _{Z \in S} \max _{u \in \mathbb{R}^{m}}\left\{g^{*}\left(x^{*}\right)-f^{*}(u)-\operatorname{Tr}\left[Z F_{0}\right]-\delta_{C}^{*}\left(x^{*}-u+\hat{F}^{*}(Z)\right)\right\}\right\} .
\end{aligned}
$$

Since $\inf (S D P)=\inf (S D P 1),(27)$ has been proved.

Similarly, it follows from Theorem 4.2 that

$$
\inf (S D P 1)=\inf _{x^{*} \in X^{*}} \max _{Z \in S^{+}}\left\{g^{*}\left(x^{*}\right)-\left(f-Z F+\delta_{C}\right)^{*}\left(x^{*}\right)\right\}
$$

Since $Z F($.$) is continuous (Z \in S)$, for each $x^{*} \in X^{*}=\mathbb{R}^{m}$ we have that

$$
\begin{aligned}
\left(f-Z F+\delta_{C}\right)^{*}\left(x^{*}\right) & =\min _{u \in \mathbb{R}^{m}}\left\{\left(f+\delta_{C}\right)^{*}\left(x^{*}-u\right)+(-Z F)^{*}(u)\right\} \\
& =\left(f+\delta_{C}\right)^{*}\left(x^{*}+\hat{F}^{*}(Z)\right)+\operatorname{Tr}\left[Z F_{0}\right] .
\end{aligned}
$$

The equality (28) now follows from (29) and (30).

Corollary 7.1 If $C=X$ and the set $\bigcup_{Z \in S, r \geq 0}\left(-\hat{F}^{*}(Z), \operatorname{Tr}\left[Z F_{0}\right]+r\right)$ is closed, then

$$
\inf (S D P)=\inf _{x^{*} \in \mathbb{R}^{m}}\left\{\max _{Z \in S}\left\{g^{*}\left(x^{*}\right)-f^{*}\left(x^{*}+\hat{F}^{*}(Z)\right)-\operatorname{Tr}\left[Z F_{0}\right]\right\}\right\} .
$$

We now give an optimality condition for (SDP).

Theorem 7.2 For the Problem (SDP), assume that $\sigma^{*}$ is (FM). Then, $\bar{x} \in A$ is a global solution of (SDP) if and only if for each $\epsilon \geq 0$ and each $x^{*} \in \partial_{\epsilon} g(\bar{x})$, there exist $Z \in S$ and $\epsilon_{1}, \epsilon_{2} \geq 0$ such that $\epsilon_{1}+\epsilon_{2}=\epsilon-\operatorname{Tr}[Z F(\bar{x})]$ and

$$
x^{*}+\hat{F}^{*}(Z) \in \partial_{\epsilon_{1}} f(\bar{x})+N_{\epsilon_{2}}(C, \bar{x}) .
$$

In particular, if $\bar{x} \in A$ is a global solution of (SDP), then for each $x^{*} \in \partial g(\bar{x})$ there exists $Z \in S$ such that

$$
x^{*}+\hat{F}^{*}(Z) \in \partial f(\bar{x})+N(C, \bar{x}) \text { and } \operatorname{Tr}[Z F(\bar{x})]=0 .
$$


Proof. We first observe that since $f$ is continuous, the condition (CC) holds. Suppose that $\bar{x}$ is a global solution of (SDP). Then, by Theorem 6.1, for each $\epsilon \geq 0$ and each $x^{*} \in \partial_{\epsilon} g(\bar{x})$, there exist $Z \in S^{+}$and $\epsilon_{1}^{\prime}, \epsilon_{2}^{\prime}, \epsilon_{3}^{\prime} \geq 0$ such that $\epsilon_{1}^{\prime}+\epsilon_{2}^{\prime}+\epsilon_{3}^{\prime}=\epsilon-\operatorname{Tr}[Z F(\bar{x})]$ and

$$
\left.x^{*} \in \partial_{\epsilon_{1}^{\prime}} f(\bar{x})+N_{\epsilon_{2}^{\prime}}(C, \bar{x})\right)+\partial_{\epsilon_{3}^{\prime}}(Z, F(\bar{x})) .
$$

Note that $\partial_{\epsilon_{3}^{\prime}}(-Z F(\bar{x}))=\partial(-Z F(\bar{x}))=-\hat{F}^{*}(Z)$ and that $\partial_{\epsilon_{1}^{\prime}} f(\bar{x}) \subset \partial_{\epsilon_{1}^{\prime}+\epsilon_{3}^{\prime}} f(\bar{x})$. So, if we set $\epsilon_{1}=\epsilon_{1}^{\prime}+\epsilon_{3}^{\prime}$, and $\epsilon_{2}=\epsilon_{2}^{\prime}$, then it comes that

$$
x^{*} \in \partial_{\epsilon_{1}} f(\bar{x})+N_{\epsilon_{2}}(C, \bar{x})-\hat{F}^{*}(Z) \text { and } \epsilon_{1}+\epsilon_{2}=\epsilon-\operatorname{Tr}[Z F(\bar{x})] .
$$

The necessary condition is proved. The sufficiency condition follows from Theorem 6.1 and a from similar argument the previous one.

When $g \equiv 0$, Problem (SDP) becomes the convex semi-definite problem of the form

$$
\begin{array}{lll}
\text { (CSDP) } & \inf & f(x) \\
\text { subject to } & x \in C, F_{0}+\sum_{i=1}^{m} x_{i} F_{i} \succeq 0
\end{array}
$$

Corollary 7.2 Assume that $\sigma^{*}$ is (FM). Then

$$
\begin{aligned}
& \text { (i) } \quad \inf (C S D P)=\max _{Z \in S} \max _{u \in \mathbb{R}^{m}}\left\{-f^{*}(u)-\delta_{C}^{*}\left(-u+\hat{F}^{*}(Z)\right)-\operatorname{Tr}\left[Z F_{0}\right]\right\} . \\
& \text { (ii) } \inf (C S D P)=\max _{Z \in S} \inf _{x \in C}\{f(x)-\operatorname{Tr}[Z F(x)]\} .
\end{aligned}
$$

Proof. We give a proof for (ii) only. The proof of the equality (i) is quite similar. Taking $g \equiv 0$ in Theorem 7.1, we get that

$$
\begin{aligned}
\inf (C S D P) & =\max _{Z \in S}\left\{-\left(f+\delta_{C}\right)^{*}\left(\hat{F}^{*}(Z)\right)-\operatorname{Tr}\left[Z F_{0}\right]\right\} \\
& =\max _{Z \in S}\left\{\inf _{x \in X}\left(f+\delta_{C}\right)(x)-\left(\hat{F}^{*}(Z), x\right)-\operatorname{Tr}\left[Z F_{0}\right]\right\} \\
& =\max _{Z \in S} \inf _{x \in C}\left\{f(x)-\sum_{1 \leq i \leq m} x_{i} \operatorname{Tr}\left[Z F_{i}\right]-\operatorname{Tr}\left[Z F_{0}\right]\right\} \\
& =\max _{Z \in S} \inf _{x \in C}\{f(x)-\operatorname{Tr}[Z F(x)]\} .
\end{aligned}
$$

The proof is complete.

The following corollary gives an optimality condition for (CSDP), which is a direct consequence of Theorem 7.2 and hence, its proof will be omitted.

Corollary 7.3 Assume that $\sigma^{*}$ is (FM), then a point $\bar{x} \in A$ is a solution of (CSDP) if and only if there exists $Z \in S$ such that

$$
\hat{F}^{*}(Z) \in \partial f(\bar{x})+N(C, \bar{x}), \quad \operatorname{Tr}[Z F(\bar{x})]=0 .
$$

Note that the assertion (ii) in Corollary 7.2 is nothing else than the strong Lagrange duality for the problem (CSDP) and the optimality condition given in Corollary 7.3 was established in [17] (Corollary 4.1) where $C=X$.

\section{References}

[1] Amahroq T., Penot J.P., Syam A.: Local minimization of the difference of two functions, Preprint of the Laboratory of Applied Mathematics of Pau (LMA), 0531 (2005)

[2] Amara M., Obeid A., Vallet G.: Existence results for a degenerated nonlinear elliptic partial differential equation, J. Math. Anal. Appl., 310, 641-656 (2005) 
[3] An L.T.H.: An efficient algorithm for globally minimizing a quadratic function under convex quadratic constraints, Math. Program., 87(A) 401-426 (2000)

[4] An L.T.H., Tao P.D.: The DC (difference of convex functions) programming and DCA revisited with DC models of real world non-convex optimization problems, Ann. Oper. Res., 133, 23-46 (2005)

[5] An L.T.H., Tao P.D., Hao D.N.: Solving an inverse problem for an elliptic equation by d.c. programming, J. Glob. Optim., 25, 407-423 (2005)

[6] Ben-Tal A.B., Nemirovski A.: Lectures on modern convex optimization: Analysis, algorithms, and engineering applications, SIAM-MPS, Philadelphia (2001)

[7] Bot R.I., Kassay G., Wanka G.; Strong duality for generalized convex optimization problems, J. Optimization Theory Appl., 127, 45-70 (2005)

[8] Bot R.I., Wanka G.: Frakas-type results with conjugate functions, SIAM J. Optim., 15, 540-554 (2005)

[9] Bot R.I., Wanka G.: An alternative formulation for a new closed cone constraint qualification, Nonlinear Anal.: Theory, Methods, Appl., 64, 1367-1381 (2006)

[10] Boyd S., Vandenberghe L.: Convex optimization, Cambridge University Press, New York (2004)

[11] Burachik R.S., Jeyakumar V.: Dual condition for the convex subdifferential sum formula with applications, J. Convex Anal., 15, 540-554 (2005)

[12] Burachik R.S., Jeyakumar V.: A new geometric condition for Fenchel's duality in infinite dimensional spaces, Math. Program. 104(B) 229-233 (2005).

[13] Burachik R.S., Jeyakumar V., Wu Z.-Y.: Necessary and sufficient conditions for stable conjugate duality, Nonlinear Anal.: Theory, Methods, Appl., 64 1998-2006 (2006).

[14] Combari C., Laghdir M., Thibault L.: Sous-différentiels de fonctions convexes composées, Ann. Sci. Math. Qué, 18, 119-148 (1994)

[15] Dinh N., Goberna M.A., López M.A.: From linear to convex systems: consistency, Farkas' lemma and applications, J. Convex Anal., 13, 113-133 (2006)

[16] Dinh N., Goberna M.A., López M.A., Son T.Q.: New Farkas-type results with applications to convex infinite programming, ESAIM Control Optim. Calc. Var. (to appear)

[17] Dinh N., Jeyakumar V., Lee G.M.: Sequential Lagrangian conditions for convex programs with applications to semi-definite programming, J. Optimization Theory Appl., 125, 85-112 (2005)

[18] Dinh N., Vallet G., Nghia T.T.A.: Generalized Farkas lemmas for systems involving convex and DC functions and its applications, Preprint of the Laboratory of Applied Mathematics of Pau (LMA), 0621 (2006)

[19] Hiriart-Urruty J. B.: $\epsilon$-Subdifferential, in Convex Analysis and Optimization, Edited by J. P. Aubin and R. Vinter, Pitman, London, England, pp. 43-92 (1982)

[20] Hiriart-Urruty J. B.: From convex optimization to non-convex optimization necessary and sufficient conditions for global optimality, in from convexity to non-convexity, Edited by R.P. Gilbert, P.D. Panagiotopoulos, and P.M. Pardalos, Kluwer Academic Publisher, London, pp. 219-239 (2001)

[21] Horst R., Thoai N.V.: DC programming: overview, J. Optimization Theory Appl., 103, 1-43 (1999)

[22] Jeyakumar V.: Asymptotic dual conditions characterizing optimality for convex programs, J. Optimization Theory Appl., 93, 153-165 (1997) 
[23] Jeyakumar V., Dinh N., Lee G.M.: A new closed cone constraint qualification for convex Optimization, Applied Mathematics Research Report AMR04/8, School of Mathematics, University of New South Wales, (2004)

[24] Jeyakumar V., Lee G. M., Dinh N.: New sequential Lagrange multiplier conditions characterizing optimality without constraint qualifications for convex programs, SIAM J. Optim., 14, 534-547 (2003)

[25] Jeyakumar V., Rubinov A. M., Glover B. M., Ishizuka Y.: Inequality systems and global optimization, J. Math. Anal. Appl., 202, 900-919 (1996)

[26] Jeyakumar V., Song W., Dinh N., Lee G. M.: Stable strongly duality in convex optimization, Applied Mathematics Research Report AMR05/22, School of Mathematics, University of New South Wales (2005)

[27] Jeyakumar V., Wu Z.Y., Lee G. M., Dinh N.: Liberating the subgradient optimality conditions from constraint qualifications, J. Glob. Optim., 36, 127-137 (2006)

[28] Laghdir M.: Optimality conditions and Toland's duality for a non-convex minimization problem, Mat. Versn., 55, 21-30 (2003)

[29] Martiez-Legaz J-E, Volle M.: Duality in D.C. programming: The case of several D.C. constraints, J. Math. Anal. Appl., 237, 657-671 (1999)

[30] Ramana M.V., Tucel L., Wolewicz H.: Strong duality for semi-definite programming, SIAM J. Optim., 7, 644-662 (1997)

[31] Rockafellar R.T., Wets R.J-B.: Variational analysis, Springer, Heidelberg (1998)

[32] Toland J.F.: Duality in non-convex optimization, J. Math. Anal. Appl., 66, 399-415 (1978)

[33] Tuy H.: A note on necessary and sufficient condition for global optimality, preprint, Institute of Mathematics, Hanoi (1989)

[34] Tuy H.: Convex analysis and global optimization, Kluwer Academic Publishers, Dordrecht (1998)

[35] Tuy H., Oettli W.: On necessary and sufficient conditions for global optimality, Rev. Mat. Apl., 15, 39-41 (1994)

[36] Yuille A.L., Rangarajan A.: The concave-convex procedure, Neural Comput., 15, 915-936 (2003)

[37] Zalinescu C.: Convex analysis in general vector spaces, World Scientific, Singapore (2002) 